\title{
The outcome of first metatarsophalangeal joint arthrodesis using a locking compression plate
}

\author{
Meijer JG' ${ }^{1}$, Gräbe $J^{2}{ }^{2}$, Greyling P $P^{3}$ \\ 1 MBChB(Pret); Orthopaedic registrar, Department of Orthopaedics, Kalafong Hospital, University of Pretoria, Pretoria, South Africa \\ 2 MBChB(Pret), FCS Orth(SA), MMed Orth(Pret); Orthopaedic surgeon, Department of Orthopaedics, Steve Biko Academic Hospital, \\ University of Pretoria, Pretoria, South Africa \\ 3 MBChB(Pret), FCS Orth(SA), MMed Orth(Pret); Orthopaedic surgeon, Department of Orthopaedics, Steve Biko Academic Hospital, \\ University of Pretoria, Pretoria, South Africa
}

Corresponding author: Dr JG Meijer, 165C Lys Street, Rietondale, 0084; tel: +27 (12) 318 6400; email: jansiemeijer@gmail.com

\begin{abstract}
Background: Arthrodesis of the first metatarsophalangeal joint (MTPJ) is a common and frequently performed procedure in the practice of orthopaedic foot and ankle surgery. Fusion techniques and preferred surgical implants have significantly evolved during recent years. It is however still under debate which surgical device provides the best outcome. One of the modern techniques described includes the use of a dorsal anatomical locking plate. These plates are usually used in combination with an additional compression cross screw across the arthrodesis site. The aim of this study was to assess the outcome of arthrodesis of the first MTPJ using a dorsal locking plate without making use of additional compression cross screw fixation.

Methods: We retrospectively assessed data at an orthopaedic practice specialising in foot and ankle surgery. All patients who had a first MTPJ arthrodesis with an anatomical locking plate system between 2010 and 2016 were identified. No additional compression cross screw fixation was done in any of these cases. Standard standing dorsoplantar and lateral X-rays of the foot were taken six weeks after surgery. As a primary objective, these radiographs were assessed to determine the rate of radiological union. As a secondary objective, any other complications that occurred in the post-operative period were recorded.

Results: We included 115 patients in the study. Fifteen of these patients underwent bilateral first MTPJ arthrodesis surgery, making the total number of feet included in the study 130 . Of these, $86 \%(n=99)$ were female and $14 \%(n=16)$ were male. The mean age at the time of surgery was 54.7 years (range 37-74). An observed radiological union rate of $97 \%$ at three months after surgery was recorded. A total of four cases (3\%) presented with symptomatic non-union. Two of these were successfully revised and progressed to union before the nine-month follow-up. One patient had bilateral surgery for severe rheumatoid arthritis with poor bone quality. No union was achieved even after a revision procedure with bone grafting. A Keller-type resection arthroplasty was eventually performed in this patient. Another secondary complication that was recorded is an overall infection rate of $2 \%$.
\end{abstract}

Conclusion: The results of this retrospective study suggest that high union rates and a low incidence of complications can be expected when fusing the first MTPJ with the use of a locking plate system without the need for an additional compression cross screw.

Level of evidence: Level 4

Keywords: first metatarsophalangeal joint, fusion, arthrodesis, locking plate, union

Citation: Meijer JG, Gräbe JC, Greyling P. The outcome of first metatarsophalangeal joint arthrodesis using a locking compression plate. SA Orthop J 2020;19(4):218-222. http://dx.doi.org/10.17159/2309-8309/2020/v19n4a4

Editor: Prof. N Saragas, University of the Witwatersrand, Johannesburg, South Africa

Received: March 2020

Accepted: April 2020

Published: November 2020

Copyright: $\odot 2020$ Meijer JG. This is an open-access article distributed under the terms of the Creative Commons Attribution Licence, which permits unrestricted use, distribution and reproduction in any medium, provided the original author and source are credited.

Funding: No funding has been received for this manuscript.

Conflict of interest: The authors declare they have no conflicts of interest that are directly or indirectly related to the research. 


\section{Introduction}

Arthrodesis of the first metatarsophalangeal joint (MTPJ) is a relatively common and frequently performed surgical procedure in the practice of orthopaedic foot and ankle surgery. It was first described by Clutton in 1894 for the treatment of severe hallux valgus. ${ }^{1,2}$

A wide variety of conditions may affect the first ray of the foot, ultimately leading to significant pain and deformity of the first MTPJ. In these conditions fusion of the joint has proven to be a successful procedure. Common indications include hallux valgus, hallux rigidus, inflammatory arthropathy, salvage procedures after failed previous surgery and neuromuscular conditions..$^{2-6}$

As noted in the literature, successful first MTPJ arthrodesis has been attained with the use of a wide variety of implants ranging from crossed Kirschner wires, cerclage wiring, sutures, staples, axially or obliquely placed compression screws, intramedullary Steinman pins, external compression clamps, and dorsal plate and screw constructs. Most of these methods have demonstrated successful union rates ranging from $70 \%$ to $100 \%$. $^{3,4,6,7}$

A review of the available biomechanical studies in the literature shows that fixation using a lag screw and a dorsal plate construct has the best overall mechanical stability., Small fragment compression plates were frequently used in the past, but because of their size and prominence often caused local irritation and pressure symptoms. Because of this complication, a high rate of hardware removal was seen..$^{10,11}$ This has led to an evolution of the dorsal plate. More recently, anatomically contoured plates with locking and nonlocking screw options became available. These locking plates have a low profile and are pre-contoured to match the anatomy of the patient better, therefore aiming to negate the pressure symptoms as previously experienced with more bulky constructs.

With the advances of these anatomical locking plates it is expected that outcomes with regard to union rate and patient satisfaction should also improve. Currently these plate-and-screw constructs are mostly being used in conjunction with a compression cross screw across the arthrodesis site. ${ }^{3-5,11}$ As these constructs are biomechanically more rigid than previously used implants, the question arises whether an additional compression cross screw is still necessary in order to achieve an adequate union rate when fusing the first MTPJ.

The purpose of this study was to assess the outcome of arthrodesis of the first MTPJ using a dorsal locking plate without making use of additional compression cross screw fixation.

\section{Materials and methods}

This study was conducted at an orthopaedic practice specialising in foot and ankle surgery. Ethical approval was obtained from the Research Ethics Committee of the University of Pretoria prior to the commencement of data collection. The data of all patients who underwent first MTPJ arthrodesis with a dorsal anatomical locking plate between January 2010 and December 2016 were retrospectively reviewed. Patients were included if they were above 18 years and underwent first MTPJ arthrodesis with a dorsal locking plate without additional compression cross screw fixation. Patients were excluded in the case of revision arthrodesis surgery and if any device other than a dorsal locking plate in isolation was used.

\section{Surgical procedure}

All patients were operated by the same surgeon (senior author, JCG). The same surgical technique was consistently used in all cases. An above-ankle tourniquet was applied and inflated to $250 \mathrm{mmHg}$. The first MTPJ was accessed through a standard dorsal approach. Joint surface preparation was done with spherical
MTPJ reamers. All cartilage was removed in order to achieve congruent cancellous surfaces in a concave-convex configuration. Multiple small holes were drilled into both surfaces with a $1.9 \mathrm{~mm}$ drill bit until adequate bleeding was observed. To determine the correct fusion position, we simulated weightbearing by applying a flat surface to the sole of the foot. We used either the flat bottom area of a large kidney dish or the cover of the plate set. We aimed at a position of slight valgus and slight dorsiflexion of the big toe. There had to be a space of approximately 8-10 mm between the flat surface and the plantar skin of the proximal phalanx. The MTPJ was then temporarily stabilised with one Kirschner wire, and a titanium dorsal locking plate (Acumed ${ }^{\circledR}$, Oregon, USA) was applied. The same plate was used in all cases and had the following specifications: $10^{\circ}$ of lateral angulation (hallux valgus angle) and a $4^{\circ}$ dorsiflexion angle. For some of the cases, the plate had to be manually bent to either increase or decrease the dorsiflexion angle. This adjustment depended on the alignment of the foot, where slightly more dorsiflexion was required for a cavus foot and a lower dorsiflexion angle was preferred for a flat foot. First, the two distal screws were inserted into the proximal phalanx. Manual compression was applied and a compression screw was then inserted into the metatarsal shaft. Post-operatively all patients were placed in a protective shoe for six weeks. Heel weight-bearing was allowed as tolerated. Wounds were inspected and sutures were removed two weeks after surgery.

Standard standing dorsoplantar and lateral foot radiographs were reviewed to assess the rate of radiological union. X-rays were routinely taken six weeks after surgery. In most patients there were already signs of union at six weeks. If union was not evident at six weeks, we repeated $X$-rays at three months and again at six months if there was still any doubt. A cut-off of six months was used to allow for timely intervention in cases of symptomatic non-union. Union was defined as the presence of osseous trabeculation across the joint interface involving at least three of the four bridging cortices

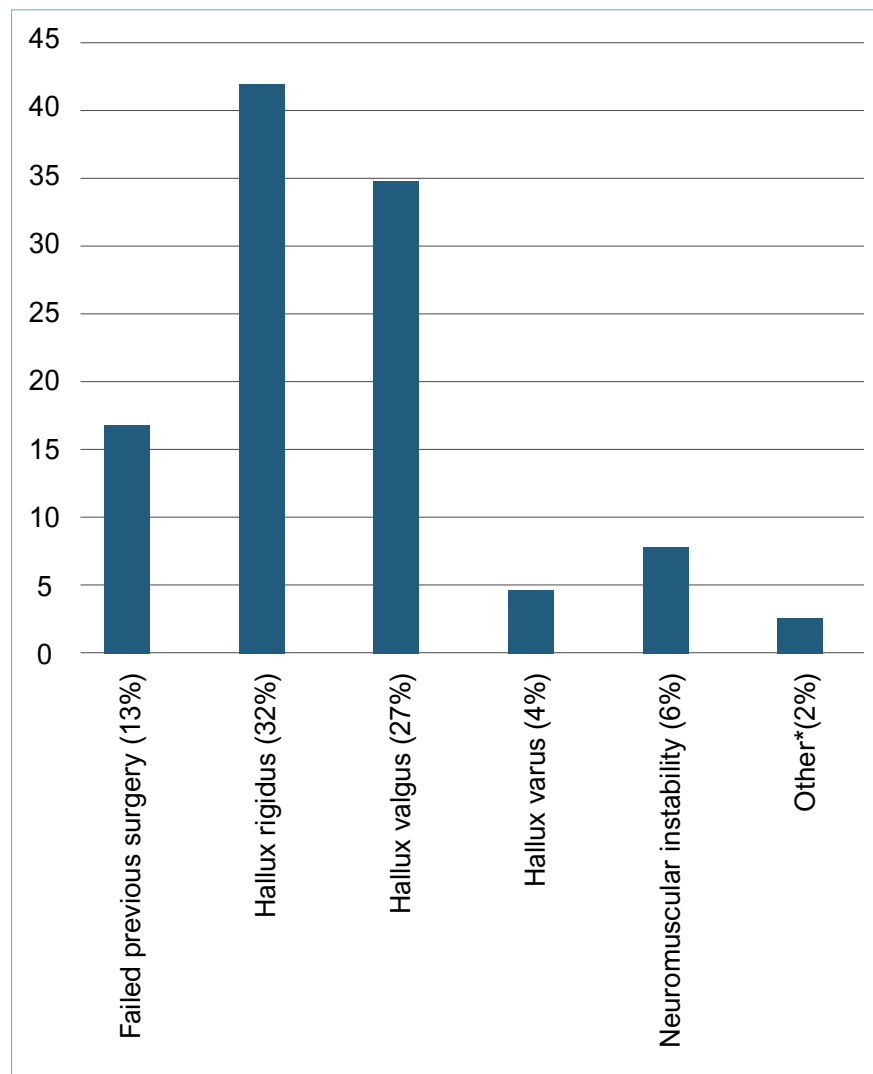

Figure 1. Number of cases performed per surgical indication *Hallux flexus, osteochondral lesions and post-traumatic arthritis 
(medial and lateral cortices on the dorsoplantar view and superior and inferior cortices on the lateral view). ${ }^{12}$ Radiological analysis was done by two experienced orthopaedic foot and ankle surgeons (senior authors JCG and PG).

General complications other than non-union were also recorded. These mainly included the prevalence of post-operative wound problems and infection. The different indications for arthrodesis were documented as shown in Figure 1.

\section{Statistical analysis}

Continuous variables were described using mean with a range. Categorical variables were described using frequency and proportions. The rate of complications was expressed as a proportion of all cases with a 95\% confidence interval. All analyses were conducted in Excel 2013.

\section{Results}

A total of 115 patients were included in the study. Fifteen of these patients had bilateral first MTPJ arthrodesis surgery, making the total number of feet included in the study 130.

The study population consisted of 99 females (86\%) and 16 males (14\%). Fourteen of the 15 patients who underwent bilateral first MTPJ arthrodesis were female. These cases were predominantly performed for severe hallux valgus in patients with rheumatoid arthritis or neuromuscular conditions. The mean age at the time of surgery was 54.7 years (range $37-74$ ).

The majority of cases were performed for hallux rigidus $(n=42$; $32 \%)$. A total of 35 surgeries (27\%) were done for hallux valgus and $17(13 \%)$ for failed previous surgery of the hallux. Of the 20 surgeries performed for inflammatory arthropathy, rheumatoid arthritis was the most common indication $(n=17)$. Other indications in this group were systemic lupus erythematosus, gout and psoriasis. Eight cases were done for neuromuscular conditions $(6 \%)$ and five cases for hallux varus $(4 \%)$. The remaining cases were performed for hallux flexus, post-traumatic deformity with secondary joint degeneration and osteochondral lesions (Figure 1).

A total of seven complications in six patients were recorded, making the overall complication rate $5 \%(95 \% \mathrm{Cl}=2-9 \%)$. Each complication in relation to the initial indication for first MTPJ arthrodesis is outlined in Table I.

An overall union rate of $97 \%$ was recorded. A total of 126 out of the 130 cases progressed to radiological union by the threemonth follow-up visit. Four cases in three patients had evidence of radiological non-union, making the overall non-union rate $3 \%(95 \% \mathrm{Cl} 0.1-6.0 \%)$. Of these patients, one was operated for hallux rigidus and another for neuromuscular deformity. The third patient developed non-union in both feet following bilateral first MTPJ arthrodesis for rheumatoid arthritis. All three patients presented with a symptomatic non-union. Two of these cases (hallux rigidus and neuromuscular deformity) were revised with the additional use of bone graft and progressed to radiological union within nine months of the index surgery. A cross screw was not

Table I: Surgical indications in relation to specific complications

\begin{tabular}{|l|c|c|}
\hline \multicolumn{1}{|c|}{ Indication for surgery } & Non-union & Infection \\
\hline Hallux rigidus & 1 & 0 \\
\hline Rheumatoid arthritis & 2 & 0 \\
\hline Neuromuscular conditions & 1 & 0 \\
\hline Failed previous surgery & 0 & 2 \\
\hline Hallux valgus \& Charcot arthropathy & 0 & 1 \\
\hline Total & $\mathbf{4}$ & $\mathbf{3}$ \\
\hline
\end{tabular}

used in the revision surgeries. The bone quality of the patient with rheumatoid arthritis was too soft and we thought that an additional screw would not have given any more stability. A different plate was used due to the bone destruction of her proximal phalanges. The revision surgery of this rheumatoid patient also failed, and she was eventually revised to a Keller-type procedure in both feet

The rate of infection in our study was $2 \%(95 \% \mathrm{Cl} 0.3-4.9 \%)$. Three patients presented with infection in the post-operative period. Arthrodesis in these cases was performed for two patients who had previously failed surgery and for one diabetic patient with severe hallux valgus and Charcot arthropathy. The diabetic patient presented with infection nine weeks after surgery and the other two patients at 10 and 14 weeks. The stability of the fusions was evaluated clinically and radiologically, and all three cases were found to be solidly united. Successful surgical debridement, hardware removal and antibiotic cover was done in each case, resulting in complete resolution of infection at the six-month followup. No secondary surgeries were required after debridement and hardware removal in all three patients.

\section{Discussion}

Arthrodesis is defined as the surgical fusion of a diseased joint for the purposes of obtaining pain relief and stability. ${ }^{13}$ The aim of first MTPJ arthrodesis is to achieve an immobile, pain-free joint that contributes to forefoot stability, improved gait and, ultimately, an improvement in quality of daily living. ${ }^{6,14}$ This procedure is frequently performed in the practice of orthopaedic foot and ankle surgery and has a multitude of indications. Common indications include hallux valgus, hallux rigidus, inflammatory arthropathy, salvage procedures after failed previous surgery and neuromuscular conditions. ${ }^{2-6}$ Similar indications for first MTPJ arthrodesis were found in our cohort.

In the present study we assessed the outcome of first MTPJ arthrodesis with the use of a dorsal locking plate system without the use of additional compression cross screw fixation. We had a low overall complication rate of $5 \%$, consisting only of cases that included non-union and infection. This rate is in keeping with reported overall complication rates in the literature ranging between $2.6 \%$ and $13.3 \% .4,5,15$ The recorded radiological union rate in this study was $97 \%$ (126 of 130 ). This is consistent with various previous studies that have also demonstrated the reliability of arthrodesis when managing a variety of conditions affecting the first MTPJ. ${ }^{5,6,11,14,15}$ An example of a case where successful union was achieved following arthrodesis for severe hallux rigidus is shown in Figures 2 and 3.

We recorded a low infection rate of $2 \%$. This is in keeping with infection rates published in the literature. A recent study by Fazal et al. showed a $3 \%$ infection rate following first MTPJ arthrodesis in their cohort of 26 patients. ${ }^{16}$ Two of the infection cases in our study

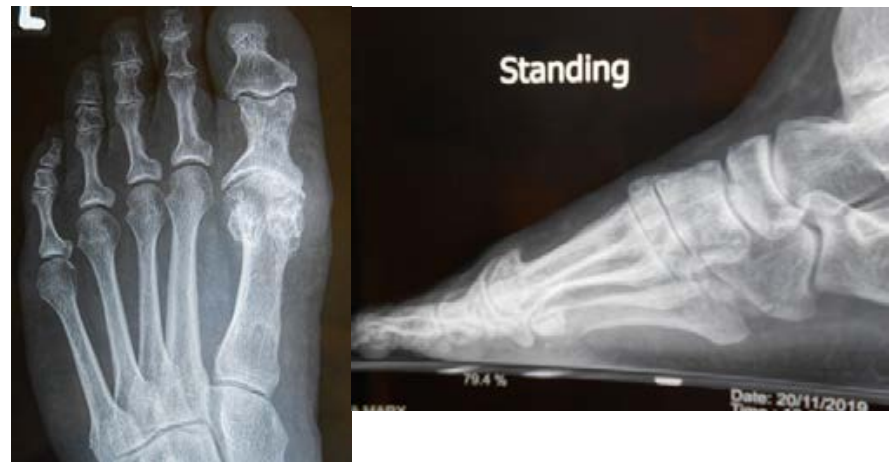

Figure 2. Pre-operative dorsoplantar and lateral X-rays showing a case of severe hallux rigidus 


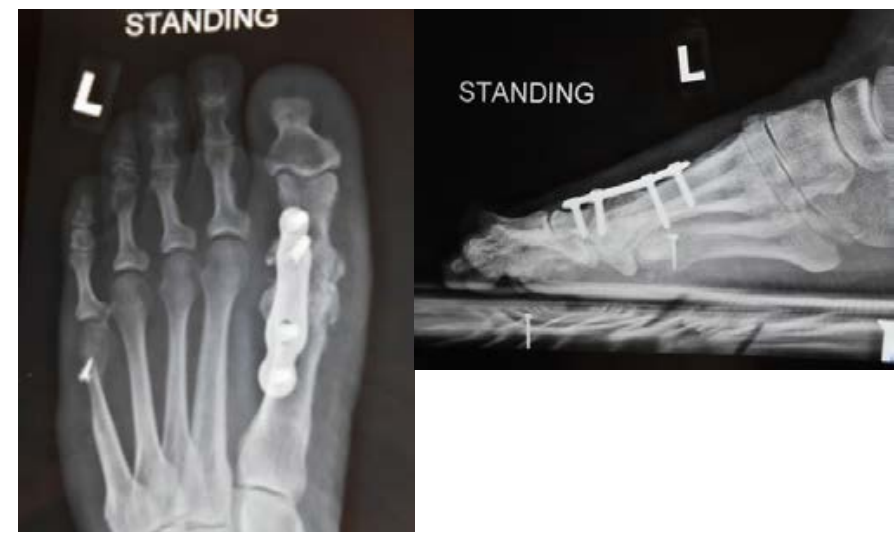

Figure 3. Post-operative X-rays showing successful radiological union

had previous failed surgery to the hallux and one had diabetes with Charcot arthropathy. These indications for arthrodesis are known to have a higher post-operative infection risk. ${ }^{17}$

A multitude of different fixation techniques have been described for arthrodesis of the first MTPJ.-6,11,14 These range from crossed Kirschner wires, cerclage wiring, sutures, staples, axially or obliquely placed compression screws, and dorsal plate and screw constructs with or without additional lag screw fixation. The most commonly used methods have demonstrated successful union rates ranging from $70 \%$ to $100 \%$ with an average of $90 \% .5,11$ The ideal fixation device should be mechanically strong, biocompatible, bone-conserving, and should be inserted with a reproducible surgical technique. It should also not require routine removal. ${ }^{11}$

In a study by Denning and Van Erve it was noted that currently the most used methods of fixation are either dorsal plates or crossed screw constructs. ${ }^{18}$ Claassen et al. compared the outcomes of fixation using either crossed screws or dorsal plates and a cross screw and found a significantly higher non-union rate in the crossed screws group. ${ }^{5}$ Biomechanical studies also indicate that a dorsal plate-and-screw construct provides greater mechanical stability and rigidity. ${ }^{8,9}$ In a study by Politi et al. it was determined that the dorsal plate and lag screw offers the biomechanically strongest fixation method for first MTPJ arthrodesis. ${ }^{9}$

There has been considerable advancement in the design of dorsal plates, with modern constructs having a lower profile and an anatomical contour that is more specific to the first MTPJ. Recent literature showed that successful union and high patient satisfaction can be expected when fusing the first MTPJ with these dorsal anatomical locking plates. ${ }^{3,4,11,12}$ This construct also allows for earlier weight-bearing and improved rehabilitation in the post-operative period. ${ }^{4}$ Younger and more active patients have also shown to benefit from this form of arthrodesis and can generally expect satisfactory results allowing them to return to a wide variety of sports and activities post-operatively. ${ }^{19}$ Flavin and Stephens reported that the combination of a low-profile contoured titanium plate with an additional cross screw and a ball-and-socket bone-end preparation had both operative and biomechanical advantages over other fixation techniques. Their patients had significant improvements in outcomes scores, union rates and other radiological parameters. ${ }^{20}$ Due to a concern that these plates may not achieve adequate compression over the fusion site, they are often used in combination with a compression cross screw. The results of the present study, however, show that a very high union rate can still be expected without the addition of a compression cross screw. This rate is comparable or even superior to the fusion rates found in studies assessing the outcomes following arthrodesis with a compression cross screw and dorsal plate..$^{3,4,11}$

In a more recent retrospective study by Cone and his colleagues, it was determined if the addition of a lag screw to a dorsal locking plate would influence union rate and final alignment after fusion. ${ }^{6}$ They noted that post-operative dorsiflexion angles were better maintained in cases where additional lag screw fixation was used, but found no statistically significant difference in the union rate when comparing fixation with a lag screw and dorsal plate to fixation with a dorsal plate in isolation. Their overall union rate, regardless of the fusion method, was $86 \%$. This is significantly lower than the $97 \%$ noted in our study.

The principle limitation of this study is its retrospective nature. It therefore depended on accurate and reliable data collection. The primary author (JGM) meticulously reviewed all data collected to address this issue. Another limitation is the fact that a control group where compression cross screws were used in conjunction with a locking plate could not be added to the study. The rate of radiological union was the main objective in this study. Other outcome measures like patient satisfaction, gait analysis and improvement of radiological parameters like dorsiflexion and hallux valgus angles were not assessed. We also did not collect and analyse outcome scores. The use of outcome scores and radiological parameters other than union rate could provide a more encompassing assessment of outcome. The large sample size is considered a strength of this study.

\section{Conclusion}

The results of this retrospective study suggest that an overall low complication rate specifically with regard to non-union and infection can be expected when fusing the first MTPJ with a dorsal locking plate system. High union rates can be expected without the need for additional compression cross screw fixation.

\section{Ethics statement}

Approval from the faculty of Health Science's Ethics committee (University of Pretoria) was obtained prior to commencement of the study (protocol number: 504/2017). Ethical principles were adhered to, as outlined by the World Medical Association Declaration of Helsinki.

\section{Declaration}

The authors declare authorship of this article and that they have followed sound scientific research practice. This research is original and does not transgress plagiarism policies

\section{Author contributions}

JGM was responsible for data capture, data analysis, first draft preparation, manuscript preparation and manuscript revision.

JCG and PG were study supervisors and responsible for study conceptualisation, manuscript preparation and manuscript revision.

\section{ORCID}

Meijer JG (D) https://orcid.org/0000-0001-7773-6081

Gräbe JC (iD http://orcid.org/0000-0003-1054-0505

Greyling P (iD) https://orcid.org/0000-0002-2535-4812

\section{References}

1. Clutton $\mathrm{HH}$. Treatment of hallux valgus. St Thomas Hosp Rep. 1894;22:1-12.

2. Mahadevan D, Korim MT, Ghosh A, et al. First metatarsophalangeal joint arthrodesis: Do joint configuration and preparation technique matter? Foot Ankle Surg. 2015;21(2):103-107.

3. Marudanayagam A, Appan SV. First metatarsophalangeal joint fusion using a Fyxis plate. J Orthop Surg. 2014;22(1):35-38.

4. Mann JJ, Moon JL, Brosky TA. Low-profile titanium plate construct for early weightbearing with first metatarsophalangeal joint arthrodesis. J Foot Ankle Surg. 2013;52(4):460-64.

5. Claassen L, Plaass C, Pastor M, et al. First metatarsophalangeal joint arthrodesis: a retrospective comparison of crossed-screws, locking and non-locking plate fixation with lag screw. Arch Bone Jt Surg. 2017:5(4):221-25. 
6. Cone B, Staggers JR, Naranje S, et al. First metatarsophalangeal joint arthrodesis: does the addition of a lag screw to a dorsal locking plate influence union rate and/or final alignment after fusion. J Foot Ankle Surg. 2018;57(2):259-63.

7. Fadel G, Rowley D, Abboud R. Hallux metatarsophalangeal joint arthrodesis: various techniques. The Foot. 2002;12:88-96.

8. Neufeld SK, Parks BG, Naseef GS, Melamed EA, Schon LC. Arthrodesis of the first metatarsophalangeal joint: a biomechanical study comparing memory compression staples, cannulated screws, and a dorsal plate. Foot Ankle Int. 2002;23(2):97-101.

9. Politi J, Hayes J, Njus G, Bennett GL, Kay DB. First metatarsalphalangeal joint arthrodesis: a biomechanical assessment of stability. Foot Ankle Int. 2003;24(4):332-37.

10. Coughlin MJ. Arthrodesis of the first metatarsophalangeal joint with minifragment plate fixation. Orthopedics. 1990;13:1037-44.

11. Latif A, Dhinsa BS, Lau B, Abbasian A. First metatarsophalangeal fusion using joint specific dorsal plate with interfragmentary screw augmentation: Clinical and radiological outcomes. Foot Ankle Surg. 2017;25(2):132-36.

12. Sung W, Kluesner AJ, Irrgang J, Burns $P$, Wukich DK. Radiographic outcomes following primary arthrodesis of the first metatarsophalangeal joint in hallux abductovalgus deformity. $J$ Foot Ankle Surg. 2010;49(5):446-51.

13. Parker L, Singh $D$. The principles of foot and ankle arthrodesis. $J$ Orthop Trauma. 2009;23(6):385-94.

14. Hamilton GA, Ford LA, Patel S. First metatarsophalangeal joint arthrodesis and revision arthrodesis. Clin Podiatr Med Surg. 2009;26(3):459-73.

15. Roukis TS. Nonunion after arthrodesis of the first metatarsalphalangeal joint: a systematic review. J Foot Ankle Surg. 2011;50(6):710-13.

16. Fazal MA, Wong JH, Rahman L. First metatarsophalangeal joint arthrodesis with two orthogonal two-hole plates. Acta Orthop Traumatol Turc. 2018;52(5):363-66.

17. Boyd J, Chmielewski R. Prevention of infection in foot and ankle surgery. Clin Podiatr Med Surg. 2019;36(1):37-58.

18. Dening J, Van Erve RH. Arthrodesis of the first metatarsophalangeal joint: a retrospective analysis of plate versus screw fixation. $J$ Foot Ankle Surg. 2012;51(2):172-75.

19. Da Cunha RJ, MacMahon A, Jones MT, et al. Return to sports and physical activities after first metatarsophalangeal joint arthrodesis in young patients. Foot Ankle Int. 2019;40(7):745-52.

20. Flavin R, Stephens MM. Arthrodesis of the first metatarsophalangeal joint using a dorsal titanium contoured plate. Foot Ankle Int. 2004;25(11):783-87. 